\title{
Analysis and fabrication of telecommunication outdoor sector antenna remote control unit.
}

\author{
O.V Jayasinghe (Author) \\ Department of Electrical \& Electronic Engineering \\ SLIIT - Sri Lanka Institute of Information Technology \\ Malabe, Sri Lanka \\ oshadavidu@gmail.com \\ DOI: $10.31364 / S C I R J / v 7 . i 5.2019 . P 0519655$ \\ http://dx.doi.org/10.31364/SCIRJ/v7.i5.2019.P0519655
}

\begin{abstract}
The operational cost for outdoor sector antenna establishing and position optimization is being reporting as a higher value since last five to six years. The larger the customer base, the higher cost for GSM network expansion. Many practical and functional drawbacks have been identified in the traditional sector antenna positioning system such as sequence mistakes in manual adjustments, high initial cost for installation, high maintenance cost and low reliability for long term operations and projects. This research emphasis the optimization of the outdoor sector antenna with the proper use of remote device controlling systems with microchip programming such as Arduino interface. A requirement occurred to find out a proper wireless system with microchip programming in order to do the adjustments in electro/mechanical interface. This research has done the automated wireless control unit for the outdoor sector antenna and the prototype was successfully installed and tested in a selected region. Eliminating the issues and problematic situations of prevailing system and make the new system more useful in order to implement it as a new sector antenna direction changing practice was the key expectation of this research.
\end{abstract}

Index Terms - Sector antenna, Arduino Technology, Global System for Mobile communication (GSM)

\section{INTRODUCTION}

Wireless communication has become one of the most effective and widely developed technologies in modern era. Said communication integration has improved in terms of voice transformation in the form of voice through telephony technology. Secondary multimedia data such as video, images and other data packages can be transmitted with the same methodology use in telecommunication industry. The faster of the industry growth the rate of requirement for infrastructure updates have been increased during last decade. Unfortunately the required improvement in supportive infrastructures for telecommunication was unable to fulfill the expectation. Industry needed high tech innovations in said sector in order to boost the progress of the industry simultaneously with the quality of the service output. Large investments have been noticed globally during last five years as the industry moved few more steps forward. However the development is somewhat below the expected margin according to the prevailing situation.

The major challenge which government and private telecommunication service providers have been faced is " lastmile" connection means the final link between the end user of the GSM connection and the network. Use of traditional copper wire to provide the last-mile connectivity has found high cost at the operational stage. Also the respective methodology was inadequate to meet the needs of the bandwidth intensive applications. Use of coaxial cables and power line communications were recognized as methods with practical limitations. Fiber optics will be the most effective technology can be used to fulfill the respective need but considered as high expensive for general use in the industry itself. Technically fiber optics can be utilized as a backbone application for many of telecommunication transmissions needs. Wireless connectivity has become most popular in such background and highly demanded by the GSM service providers around the world. Cost effectiveness, flexible for wide bandwidth links, signal speed and smoothness of the transmission can be listed as co-advantages of the methodology. Uncovered geographical regions were fully covered using the wireless connectivity as never before. Since the system was reported no errors above the tolerance limit, high number of users have been joined with the service during last decade. In USA it was reported as 20 million. The prevailing situation of using cellular technology around the globe has been reported as 50\% in North America, 60\% Western Europe, 70\% I Australia/Asia and 200\% in South America. Rapid growth of the users of wireless networks was made the entire system so busy and the bandwidth allocation was not enough to cater the demand. Finite number of frequency users have registered and obtained the license to use the allocated radio frequencies. The respective high demand was led the industry into a really troublesome and challenging stage. Required cost to obtain the remaining radio bandwidths were rapidly increased with the cost for base infrastructure. However the majority of the GSM service providers currently in service within the domestic boundary, have being finding possibilities to provide the service at a lower price according to the users' demand.

In general power and bandwidth are defined as the major requirement should be fulfilled for information transmission. The higher the transmissions rate the high power consuming and wide bandwidth usage in the medium. Wired transmission can be done independently in terms of segment links of transmission. Separate and controlled information flow can be performed for each link. Generally two main matters were identified I the GSM service providers as below. 
01. How to acquire more capacity in order to serve more customers within the high demanded regions with the appropriate cost maintaining.

02 . How to obtain relatively greater coverage in lower demanded areas in terms of reducing infrastructure and maintain cost.

When a specific region is about to exceed the general demand rate, service providing company has to re allocate the capacity for signal transmission. The definition of capacity has been stated as the quality of each links with the number of successful connections which user has received. From the consumers' point of view, he required the most possible quality of the links at a lower cost available. According to the general phenomenon in between consumer and the service provider, a system which optimizes the advantages for both ends should be developed as a solution. The ability to cover a large area at the initial stages of the links establish with a minimal infrastructure cost, will be helped to the service provider to drop the production cost down. Increased the range by using the latest technology and increased the capacity are became as the most important challenges which service providers are always concern about.

With this research project the emphasis has been done to enhance the position matrix of the outdoor sector antennas by sending a message from mobile phone. The respective message will be included all the related data such as mechanical and electrical tilt angels, azimuth dangles and other frequency information which will be used to change the position of the sector antenna.

The initial requirement was developed by Dialog Axiata Pvt Ltd in terms of modify the technical knowhow to change the direction of GSM, DSC/3G outdoor sector antenna using wireless technology. As the leading telecommunication service provider in South Asian region Dialog Axiata has more than 400 sites in the country. There were minimum three sector antennas have established in a one site Base Transceiver Station (BTS) and more than 1200 DSC/3G sector antennas were fixed on the same BTS. Outdoor sector antennas have attached generally on roof tops and individual supported towers both in urban and rural areas. The azimuth angle changing and the mechanical/electrical tilt changing have been done at special occasions such as new site optimization, customer complaints, coverage issues and low quality or week signal propagation. Common method used for do said changes is manual using man power. The prevailing practice is identified as really problematic and dangerous. Also high additional cost for transport and man power will be used for the operation. Automation introduce to the said practice had be done immediately according to the technical department of the company. Few of the aims of conducted the research are introducing a more effective, accurate and remote directional sector antenna which can be replaced the existing one, drop down the cost allocated for current antenna adjusting process, time saving, convenience of the controlling ability and the safety providing for workers.

\section{THE CURRENT BACKGROUND}

Dialog Axiata PLC is being practiced a manual sector antenna adjustment process as their main operational method for provide solution for the customers' complaints on GSM signal issues. However the manual process has been identified as troublesome and non-technical practice as it was based for several bearing issues of the direction of the antenna. Manual fixing was not strong enough to withstand the antenna against high gust loads in some geographical regions. The radiation distribution of the antenna was reported lower strength due to wrong bearing adjusting or change the position by its own after the adjustment due to the high impact of wind. The directional change of the antenna was based on the skill level of the respective worker too and that was recognized as a factor to be changed with respect to the accuracy of the operation.

\section{A. Sector Antenna}

The outdoor sector antenna is a signal transitions device which can be categorized as directional microwave antenna with a sector-shaped radiation pattern. The "Sector" means in geographical sense as a virtual circle which can be drawn by taking the antenna as the center point. The sector can be defined as angles with reference to the horizontal or vertical axis of the antenna. The respective portion of the circle will be treated as a sector which will be generated and propagate the GSM signal radiations. In general a single sector antenna can be used to cover the sector of 120 degree. Most of the cases three antennas have been attached to the same tower in order to make a full coverage of 360 degrees. General use of these sector antennas are for cellular phone signal distribution whereas in some locations it uses as other mobile communication signal distribution such as WIFI networks. The most effective range for a sector antenna is a 4 to $5 \mathrm{~km}$ radius.

The typical sector antenna is built up with RF connectors for coaxial cables also known as feed cables. The main reflector outer skin was made by aluminium in order to protect the device from high impacts and provide a proper platform for a continuous operation. Internal electronic parts have fixed with the support of fiberglass structure enables the antenna to withstand against high gust conditions. All the metallic parts and equipment are DC grounded in order to assure the protection. The long narrow structure of the antenna gives the fan shaped radiation pattern. According to the radiation pattern depicted, typical antenna used in a three-sector antenna station has got 66 degree angle of horizontal beam width means the signal strength at the \pm 33 degree is half( $3 \mathrm{~dB}$ down) from its peak value at the center. At \pm 60 degree angle it is recommended to set the antenna gain to zero or negligible level. Vertical beam width of the antenna is not wider than $15^{\circ}$ means $7.5^{\circ}$ in each direction. AM, FM and TV sector antennas will be needed line of site of sight over more miles or kilometres. Generally downward beam tilting has established in an antenna to achieve the coverage of the areas under the antenna. It will be avoided the RF interferences too. The coverage sector can be changed or modified by adjusting the mechanical or electronic tilt. 


\section{B. Mechanical Tilting and Electrical Tilting}

The efficiency of a cellular network is depended on correct configuration and adjustment of radiant system. The antenna optimization can be achieved accurate tilting and inclination of the antenna in related axis. The respective radiation beams can be direct to downwards called "Down Tilt" which can be seen more often. In very limited situations the antenna will be performed "Up Tilt" means make the antenna inclination to upward direction. When the operators needed to avoid the interference of radiations between two sectors Up Tilt will be performed which will be enabled each cells to distribute signals as desired. Radiation diagram changes can be significantly observed during the mechanical and electrical tilting with comparison of neutral position with no tilt. These respective results can be obtained when perform a detail drive test for the antenna at the location. According to the below mentioned radiation diagram the coverage area is reduced in central direction when perform the mechanical tilt while side direction coverage is increased. In electrical tilt, the coverage area have lost uniform signal reduction alone the direction of the antenna azimuth means the reduction in antenna's gain.

Generally mechanical tilt will be performed by using skilled man power at the respective location. The antenna is fixed to the tower via an alloy mountain. Adjusting the inclination means the manually relocate the position of the antenna by adjusting the fixing nuts. Additional smoothing and fine tuning means electrical tilting will be performed by adjusting the tuning knobs of the antenna which was located at the bottom surface. These adjusting knobs were internally connected to the internal circuits and equipments which enable the electronic parts to be adjusted more smoothly.
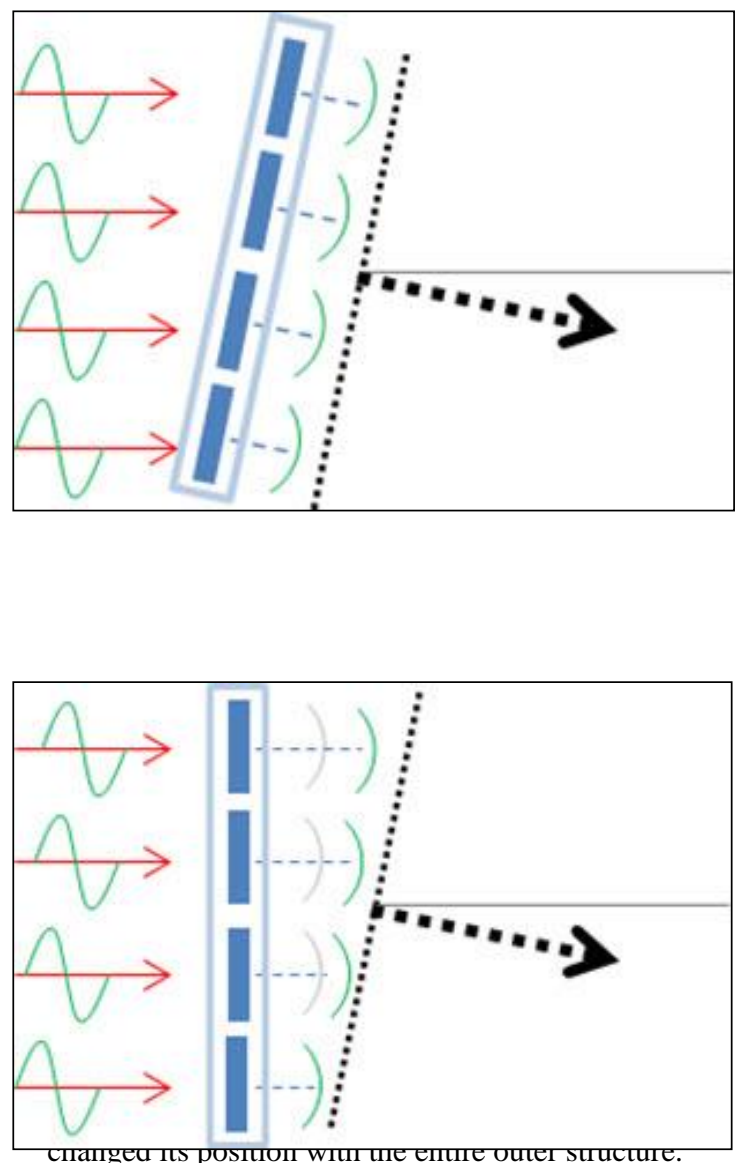

Figure 3: Electrical Tilt enables the antenna to be changed electronically as a fine tune of the signal emitting.
$\mathrm{Tl}$ Figure 4: Without Tilt and with tilt radiation patterns shows 2 tro$\mathrm{m}$ the change of radiation strength around the antenna $t$ in au accordingly mechanical and electrical tilting. has functional electronic equipment in a small physical interface. The technology was developed with a main microchip which enables to load functional instructions with $\mathrm{C}++$ programing language. Other electronic equipment such as load capacitors, resistors and transistors were installed on the board itself and run with the main set of instructions given via the IDE coding interface. Multifunctional abilities of the Arduino interface have been achieved by interconnecting the main microchip with multipurpose ICs which enables the board to be run simultaneously with microchip. The Arduino technology can be used and modified with several microprogramming technologies such as Raspberry Pi and ARM programming. 
Arduino has become one of the most popular micro programming technologies currently in service in the world. The convenient in use and ability to bridge with several other systems are main reasons for use the Arduino in many applications. Electronic extensions which developed for enhance the performances of the Arduino interface can be identified in modern automation applications. Hundreds sensors, detectors, controllers and switches can be connected to work parallel with the Arduino interface for many practical applications. Arduino hardware interface have introduced according to the purpose of the intended operation as Arduino UNO, Mega or Arduino extension board. As the Arduino and related technologies have been widely used in electromechanical innovative projects around the world, it consists with programming interface as computer software called Arduino IDE. This platform is developed with $\mathrm{C}++$ language. The code programming term as sketching. The technology is widely used in electro-mechanical innovations. The respective programming interface coupled with the computer code writing working module called Arduino IDE. The user can be loaded instructions means sketch of the coding through this software interface. The basic Arduino coding can be done using $\mathrm{C}++$ programming language. In this project, the user interface was Arduino UNO. Extensions will be worked as developing platform of basic technology and the results can be monitored using a computer interface.

\section{B. Main Features of Arduino programming}

Hardware Interface: The Aruino microchip board comes with several supportive ports and connector which enables other devices to be connected with the parent board itself. The other electronic sensors, extensions, equipment or wiring cables must be attached to the Arduino board in standard manner. It is recommended to make connection the desired electronic tools and equipments with the main Arduino board on a bread board prior to the original circuit take place. Proper connectivity should be managed by the user during the initial trial sessions to avoid the chances to overload the given voltage. The input and output pins of the main microchip of the board can be changed accordingly to the purpose of use of the Arduino. Standard practices should be maintained in the finalizing stages as the board should be controlled in the safety region of the voltage and protect from the high voltage flow across the microchip.

Sketching: Write the respective programming according to the intended output named as "Sketching" in Arduino technology. The programming codes will be written using $\mathrm{C}++$ programming language. The respective sketch should be in a standard pattern and called to the exact electronic function or supportive device to be activated in a right time. The functional instructions and timing with delays will be setup in the sketching and the simultaneous operations can be handled via a proper sketch. Commands for the connected extensions with the main board will be separated by the microchip via its pins. The pin numbers will be used to call them for an expected function. Close consideration and a exact idea on the respective project must be maintained by the programmer to obtain a better Arduino sketch.
Sketch Compiling Process: Run a sketch compiling process in a compulsory part of the Arduino programming as the sketch can be subjected to many coding mistakes or malfunctioning. The compiling process will be helped to identify the error codes wrote by the programmer with the exact segment of the sketch. Hardware connection and its issues with the main board also are highlighted in the compiling process. The programmer should be smart enough to capture the issues and recall them with an accurate coding after catches by the compiler.

Sketch Uploading: The programmer can forwarded the entire instruction to the board after the sketch has been passed by the complier and it's called "Sketch Uploading". Uploading will be facilitated by the computer software interface also known as IDE. The board will be connected to the computer via a USB2.0 or 3.0 connections. The user will be notified by the IDE interface after the sketch was successfully uploaded to the board. If the connection was not detected by the computer, Control Panel setup for COM Ports can be checked and confirmed.

Power Management: Generally the Arduino will be powered by an USB connection which can be connected to any computer interface via USB ports. When the board is prepared to fit in another place with its attachments, it should be powered via an on built power socket. It was provided on the board and the external voltage should not exceed $12 \mathrm{v}$ as per the Ardunio boards' protection guidelines. Batteries can also be attached to the board as main power supply.

Feedback Monitoring: The in-built serial monitor command comes with the main features of the Arduino IDE softwarecan be used to capture the real time funnctioning feedbacks of the entire process. Variable changing, value measuring, timely changes of outputs and inputs can be taken as examples for serial monitoring. RPM calculations, time calculations, sensitivity calculations, intensity measuring and progressive calculations can be listed as examples of serial monitoring feature of the Arduino technology. Graphs presenting and simulation of the results is the most valuable feature of the

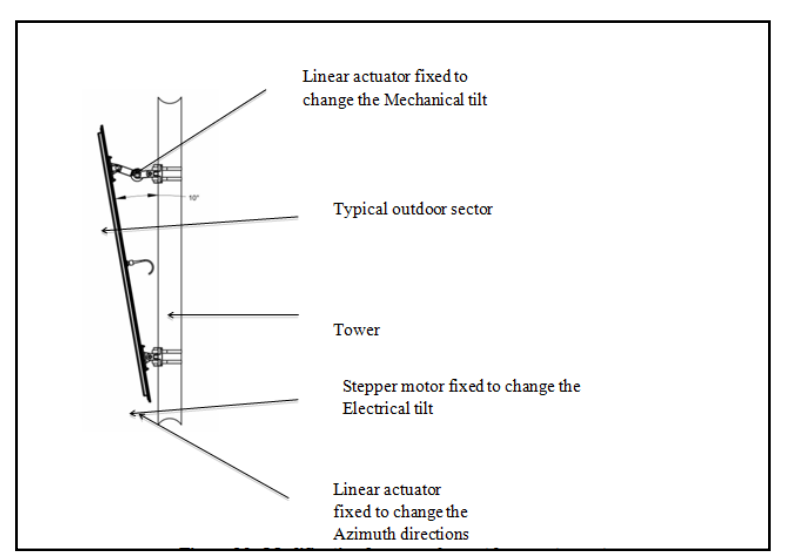

feedback monitoring of the Arduino technology. 


\section{GSM Module}

The GSM module was used to receive the GSM signals sent from the mobile form in terms of SMS. All the instructions related to the mechanical and electrical tilting were collected by the GSM module and forward to the electro-mechanical hardware interface of the sector antenna. It was built up with a GSM modem which has bridged with power supply and communication interface such as USB or RS-232. The respective module was activated and functioned with the instructions called out from the Arduino microchip interface. Specific sketch codes were used to initialize the GSM module through an Arduino program. Technical knowhow of the said GSM module bridge with the microprogramming was developed with several trial and errors. Electrical tilt was developed by connecting a remote control unit (RCU) to the manual controlling knob of the antenna. Few advantages of the GSM module can be listed as small in size and weight, lower power consumption, R\&TTE type approval, full RS232 on CMOS level with flow control, embedded TCP and tracking ability.

\section{Motor Controllers}

Moor controllers were attached to the sector antenna in terms of manoeuvring the three axis movements of the antenna. These controllers were supported to control the speed limit of the motors, stopping and initiating the rotation and RPM adjusting with forward reverse motion. These motor controllers have worked together with the microcontroller commands developed according to the numerical values forwarded from the GSM module. The motor controller type will be depended according to the performing task of the motor in the application. This project was used a voltage regulating switches as controlling switches for the motors. Voltage changes and the respective values will be sent through a microcontroller programming. Over load current protection was achieved by an over voltage protector circuit linked to the each stepper motors. Small limit motors have got in built overload protector while large stepper motors have got separate temperature sensing rely to break the line when overloading.

\section{E. L298 Motor Driver}

The L298 motor driver was used for the stepper motors as it was available in a kit which developed as user friendly electronic device. It was recognized as more reliable and high durability for such operations. L298 has got a central IC which developed for multi functions. The respective driver was built up with full bridge that can be driven up to 2 Amperes per bridge with supply voltage up to $46 \mathrm{~V}$. It can be driven two DC motors or one stepper motor, relay or solenoid. This drive is TTL compatible. Two H-Bridges of L298 can be connected in parallel to increase its current capacity to $4 \mathrm{Amp}$. It can be used in conjunction with stepper motor controller for driving one or two phase stepper motor. The respective drive is available in 15-lead multi voltage package.

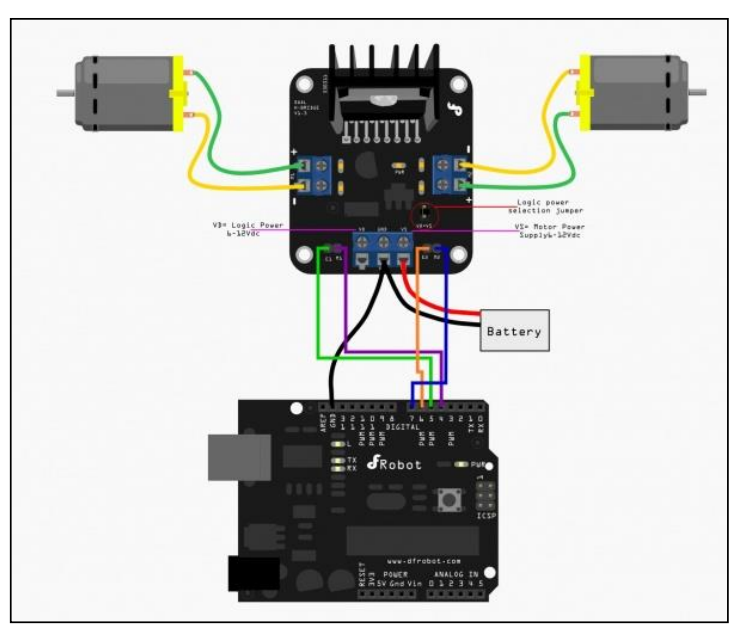

Figure 6: L298 Motor connection setup with Arduino UNO interface shows the electronic circuit needed to perform two tilting operations linked to the actuators.

\section{F. DC Gear Motors}

Geared DC motors has got built in specifications which were separated them from the general DC motors. It has got a specific gear set according to the desired RPM. The major purpose has been defined as reducing the speed of the shaft and increases the torque of the same. The series of the attached gear will be worked together during the rotation in terms of reduce the speed to a controllable figure. The respective DC motors have been used in this project to control the movements of linear actuators attached to the sector antenna outer cover.

\section{G. Linear Actuators}

Linear actuator is a device which was developed to facilitate a linear motion of any mechanically driven system. Radial motion of the conventional electric motor will be converted into a linear motion in the working principle of the actuators. Most of the cases these are used in industrial machineries and production proceedings. Hydraulic and pneumatic actuators can be seen in practical applications. Typically an electric motor is mechanically connected to rotate a lead screw. A lead screw has got a continuous helical thread machined on its circumference running along the length and this length is exactly tall with a thread on the bolt. The direction of the radial motion of the nut depends on direction of rotation of the lead screw. In this research project two linear actuators were used in terms of command driven hardware devices attached to the antenna interface.

\section{H. ATMEGA328P - AU Microcontroller}

The respective microcontroller used for the project was a low power 8 bit microcontroller. It was a self programmable and flash memory programmable device. All the 32 registers on the microcontroller are directly connected to the Arithmetic Logic Unit ( ALU) allowing two independent registers to be accessed in one single instructions executed in one clock cycle. 


\section{WorkingModule}

The working module of the proposed system is consists with three main sections as mobile phone SMS module, GSM module with pre-defined program interface and Sector antenna sensors and mechanical actuators. Initially the SMS was sent from the mobile phone which was included the directional and bearing information of the antenna to be changed. Secondly this SMS was read by the GSM module with arduino microchip interface. Finally the directions were forwarded to the antenna in the form of digital signals to change the electrical and mechanical tilt.

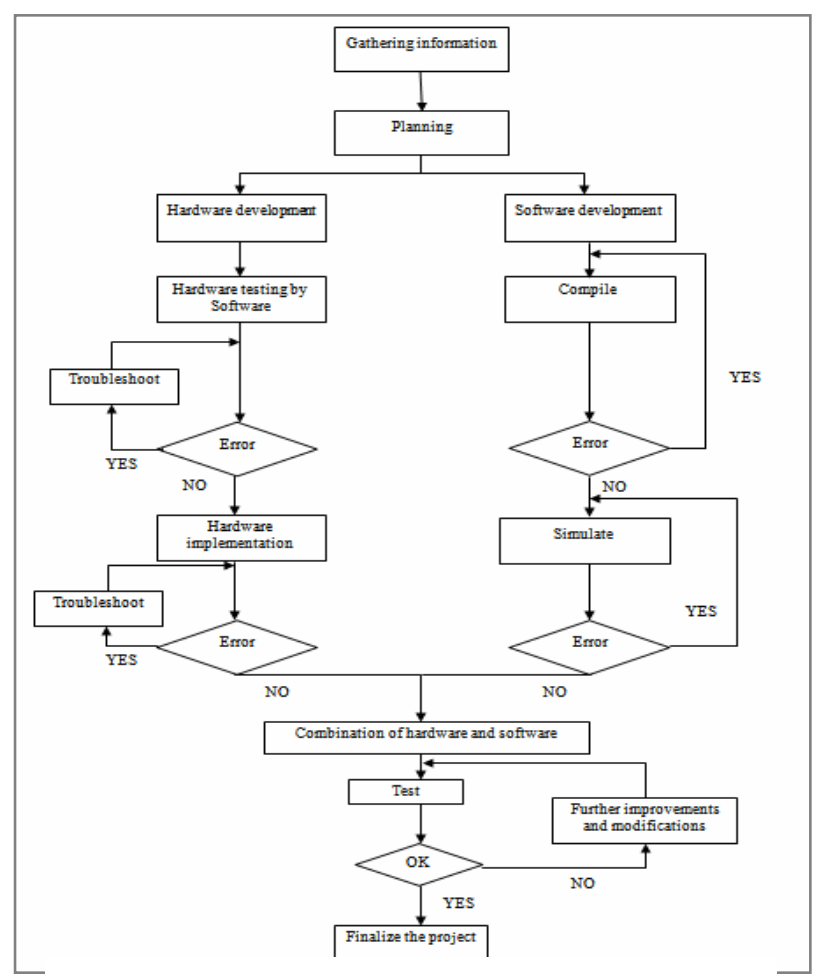

Figure 7: The flow chart of the research was developed as trial and error methodology which will be guided the process in precise measures.

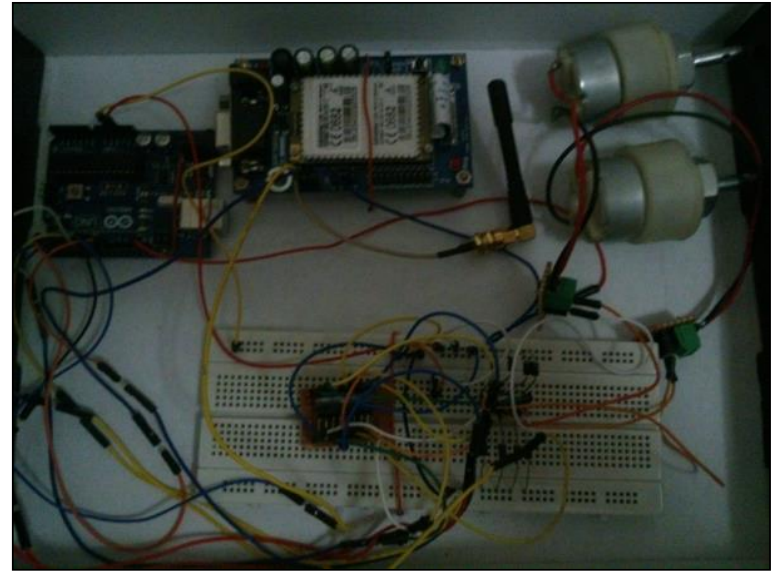

Figure 8: Initial prototype was created in terms of searching for opportunities to developments and finding the errors within the allocated technical infrastructure.

\section{B. Fabricating of Sector Antenna Mounting Module}

In order to conduct a standard mounting for newly proposed sector antenna control device, specific mounting unit have introduced during the project. This structure was modeled using Auto Cad engineering drawing software and manufactured in a workshop. Lathe operations and soldering techniques were used to manufacture the said structure using iron and aluminum as raw materials. Below are the main steps followed to complete the mounting unit of the antenna.

1. Lathe machining the cylindrical bar which enables to attach the ball bearing inside.

2. Bearing unit attached to the main bar of the mounting structure.

3. Top bearing was attached to the main bar and attached a one linear actuator which will be used to perform the mechanical tilt positioning.

4. Mounting saws and nut holes drilling operation.

5. Soldering and combining the parts.

6. Finishing and painting.

The next step was to fabricate the original electronic circuit modules which will be used in the final process of the project. PCB electronic circuit boards were used to perform the final electronic circuits as ready to fixed devices in terms of attach to the sector antenna. There were three PCB circuit boards as two L298 motor drive circuit panels and an ATMEGA 328 microcontroller PCB panel. These panels were properly supervised and tested prior to the installation. All the external parts such as actuators were combined with the electronic of the electronic circuits. GSM module, servo motors attachment and moments, actuator moments and voltage management were tested during the prototype testing. 
Figure 9: Mounting structure was made with lathe machine and other workshop operations and ensures the controllability and

\section{Fixing the Device}

The prepared unit as finalized as a one unit in the output stage. This enabled the users to mount the respective unit on the actual antenna tower without practical inconvenience. Mounting structure was designed and fabricated as user friendly model in order to eliminate real time installation issues. Current condition of the installation process has been identified as much needed practice to improve more due to the high number of incidents have reported against signal weakness. These incidents were followed by manual fixing issues done by the workers in the fields. Proper consideration on antenna attachment must be maintained during the installation process as the said antenna will be opened for heavy gust loads.

The mounting structure has been designed and fabricating such a way to obtain the maximum strength for the entire unit under any whether condition. The prototype antenna was mounted in a sample tower and tested for vibrations and middle level impacts. The results were successful and forwarded the same to the implementation stage.

\section{PROGRESS ANALYSIS}

\section{A. Results Analysis}

The respective antenna was attached and tested in a real environment as it was fixed in a regional sector antenna belongs to the Gampaha R2 regional tower. The said tower has been installed and managed by the Dialox Axiata Pvt Ltd and operational capacity reported as $30 \mathrm{Km}$ surrounding area. Signal strength of the said region has no significant issues but taken to elaborate the results as considering the convenient to access. The fixing process was supported by Dialog Axiata technical and site testing team allocated to the Gampaha regional office.

Proposed sector antenna was attached to the higher bar at the workshop and testing the working conditions and functioning ability of the device. The signal receiving and transmitting strength of the entire device was at a satisfactory level at this initial trail stage. Respective system is programmed inside the microchip of the Arduino board. GSM module was linked with the Arduino which enables the access to SMS sending and reading. During the secondary step the antenna was attached to the real location to run the test drive in real time operational condition. The initial step was to open the system for practical functions. Respective part has done by the start up SMS which has been sent to the antenna. The user must be logged in to the system by requesting permission to login. Responsive SMS was received by the user asking the username and password for the system. Sending wrong log in details in three attempts will be disconnected the login and informing the admin user by a SMS. Safety measurements have been provided to the system in such manner to eliminate the unauthorized access to the system.

During the first test run, the login details set to the Arduino programme in simple as limited letters or few numbers. After $\log$ in information passed by the system, it was automatically forwarded the user to the current bearing of the antenna. At the debut stage of the system the antenna was indicated as 0.0.0 in all three coordination axis. The adjustment setup values were started to feed to the system from this zero originates. First bearing values were feed to the antenna by SMS featured with the expected coordinating values. According to the said values in all three axis, the antenna's performances in electrical and mechanical tilting were observed. According to respective results obtained in this stage, the sector antenna was recognized as an accurate system.

\section{B. Cost Effective Advantages}

Proposed sector antenna remote control unit was recognized as the most effective method for adjusting sector antennas in terms of reducing many costs related to the real life practices. Also it has proven the effective of safety measures of the current proceedings following by the technicians. Risk of the safety of the antenna adjusting will be entirely eliminated by introducing the respective smart project. Cost allocated for the entire sector antenna adjusting and controlling was significantly reduced through the new system. Time consuming for the new project has been reduced in identical manner. Below mentioned the overall budget spent for a one unit of the proposed device. This cost has calculated for the initial process as a capital investment. Each and every time the sector antenna needed an adjustment for positioning change, the company have to spend a considerable finance resources. The comparisons of the cost reduction have proven in the second table below. 


\begin{tabular}{|c|c|c|c|}
\hline \multirow[t]{2}{*}{ Event } & \multicolumn{2}{|c|}{ Cost (USD) } & \multirow{2}{*}{$\begin{array}{l}\text { Reduction } \\
\text { in } \%\end{array}$} \\
\hline & Previous & New & \\
\hline Transport & $\begin{array}{l}28.30<\text { in } \\
\text { each time }\end{array}$ & 0 & 100 \\
\hline Labor/technicians & $\begin{array}{l}45.30<\text { in } \\
\text { each time }\end{array}$ & 0 & $100 \%$ \\
\hline Spares & $\begin{array}{l}56.60<\text { in } \\
\text { each time }\end{array}$ & $\begin{array}{l}<34 \text { Only } \\
\text { when } \\
\text { issue has } \\
\text { been } \\
\text { reported }\end{array}$ & $80 \%$ \\
\hline Maintenance & $\begin{array}{l}56.60<\text { per } \\
\text { month }\end{array}$ & $\begin{array}{l}<\quad 45.30 \\
\text { per } \\
\text { month }\end{array}$ & $30 \%$ \\
\hline Testing & $\begin{array}{l}28.30<\text { in } \\
\text { each time }\end{array}$ & $<10.20$ & $90 \%$ \\
\hline
\end{tabular}

The respective cost comparison was performed in order to accumulate the benefits related to the financial advantages. In early proceedings considerable amount of financial resources were allocated in each and every time when the repair and adjustment needed in sector antenna. Below notified all the cost related to the operational stages with the newly introduced process. It has to be proven that the proposed remote control system will reduce the existing cost significantly.

Table 2: Cost comparison with the existing process

\begin{tabular}{|c|c|c|}
\hline Product & Quantity & Price (USD) \\
\hline $\begin{array}{c}\text { Linear } \\
\text { actuators }\end{array}$ & 2 & 90.52 \\
\hline $\begin{array}{c}\text { DC Gear } \\
\text { motor }\end{array}$ & 1 & 2.83 \\
\hline $\begin{array}{c}12 \text { V/15A } \\
\text { switch mode power } \\
\text { supply }\end{array}$ & 1 & 19.80 \\
\hline $\begin{array}{c}\text { SEIMENS } \\
\text { GSM module }\end{array}$ & 1 & 31.12 \\
\hline $\begin{array}{c}\text { ATMega328P } \\
\text {-AUrocessor }\end{array}$ & 1 & 2.26 \\
\hline $\begin{array}{c}\text { L298 motor } \\
\text { driver chip }\end{array}$ & 2 & 62.23 \\
\hline $\begin{array}{c}\text { Sector antenna } \\
\text { Metal } \\
\text { mounting system }\end{array}$ & 1 & 5.66 \\
\hline PCB board & 1 & $\mathbf{2 2 2 . 9 1}$ \\
\hline $\begin{array}{c}\text { Other } \\
\text { components }\end{array}$ & - & 5.66 \\
\hline Total & 1 & \\
\hline
\end{tabular}

Conducting such calculation comparatively has shown the considerable reduction in cost allocation. This step was considered as an essential part of the project as a main requirement since it was focused on the financial advantages too. According to the results obtained it has been proven that the new automated remote control unit for the sector antennas will be converted the telecommunication providers into a remarkable turnaround.

\section{Future Developments}

This research project will be forwarded to the future improvements which can be proposed as additions to the original concept. Few of electronic phenomenon and devices can be combined and linked with the respective system as next level of the research. The respective research will be flexible enough to be worked simultaneously with several programming interfaces and electro-mechanical devices.

\section{Smart Beam Antennas}

This research can be modified with a replacement of sector antenna to the smart beam antenna instead of the existing sector antenna. The concept of using multiple antennas and innovative signal processing to serve cells more intelligently has existed for many years. In fact, varying degrees of relatively costly smart antenna systems have already been applied in defense systems. Until recent years, cost barriers have prevented their use in commercial systems. The advent of powerful low-cost Digital Signal Processors (DSPs), generalpurpose processors, as well as innovative software-based signal-processing techniques have made intelligent antennas practical for cellular communications systems. Smart beam antennas also known as adaptive array antennas, antenna arrays with smart signal processing algorithms used to identify spatial signal signature such as the direction of arrival (DOA) of the signal, and use it to calculate beam forming vectors, to track and locate the antenna beam on the mobile/target. The antenna could optionally be any sensor. Smart antenna techniques are used notably in acoustic signal processing, track and scan RADAR, radio astronomy and radio telescopes, and mostly in cellular systems like W-CDMA and UMTS. Few specifications of the smart beam antenna can be noted down as follows.

Reduce OPEX by minimizing site visits

Lower CAPEX by increasing capacity efficiency and delaying additional site builds

Enhance network quality with the new ability to optimize the beam direction and width

Experience fast, precise configuration updates

Decrease inventory costs

Prevent optimization implementation issues

Generate fewer greenhouse gases

There are up to three adjustments with Smart Beam antenna systems:

RET Remote Electrical Tilt (Tilt)

RAS Remote Azimuth Steering (Pan)

RAB Remote Azimuth Beam width (Fan)

\section{ACKNOWLEDGMENT}

In conducting this research I have received magnificent help from many quarters, which I like to put on record with great gratitude and great pleasure. 
This research was supported by Faculty of Engineering of Sri Lanka Institute of Information Technology. First and foremost I would gratefully remind Senior lecturer Dr. Lasanthe Senevirathne who gave me the guidance throughout this project with insight and expertise that greatly assisted the research as the project supervisor to share his knowledge without any hesitation.

I offer my sincere gratitude to other lecturers of Department of Electronic and Computer Engineering in Sri Lanka Institute of Information Technology for directing me in every possible ways to make this project a success and for their advices and assistance in keeping my progress on schedule. Also the lessons I have learnt during my graduate period was a major benefit in completing the research successfully.

Finally this research made possible through the help and support from family members who provide the greatest support and their encouragement throughout my studies.

\section{REFERENCES}

01. Agius A A, L. S. (2008). Intelligent Handheld Antennas for Mobile Communications Beyond the 2nd Generation". India.

02. Ahed, F., Md, S. \& Islam, S., 2016. 433

Mhz(Wireless RF) Communication between Two Arduino

UNO. American Journal of Engineering Research (AJER), 5(10), pp. 358-363.

03. Author(s): Tapan K. Sarkar, M. C.-P. (2005). Smart Antennas ( 2 ed.). Hoboken, New Jersey, USA: John Wiley \& Sons, Inc.
04. Bilgiç, M. M., Çiydem, M. \& İsenlik, T., 2011. GSM/UMTS Dual Polarization Base Station Antenna Design. IEEE, 6(2), pp. 12-21.

05. Shoukat, S., 2016. Design of a Dual Band Frequency Reconfigurable Patch Antenna for GSM and Wi-Fi Applications. IEEE, 07(3), pp. 35-42.

06. Banzi, M. (2013, November). Retrieved from ARDUINO: http://arduino.cc/en/Main/arduinoBoardUno

07. Bose, B. K. ( 2010, November). Power Electronics And Motor Drives: Advances and Trends (illustrated ed.). Burlington, USA: Academic Press.

08. Grimm, J. J. (2009). Electromagnetic Linear Actuator - Design, Manufacture and Control. Research Project, University of Southern Queensland, Queensland.

09. Hillebrand, F. (Ed.). (Wiley, 2001). GSM and UMTS: The Creation of Global Mobile Communication (illustrated, reprint ed.). Californiya, USA.

10. Hughes, A. (2005). Electric Motors and Drives: Fundamentals, Types and Applications (3 ed.). Linacre House, Jordan Hill, Oxford: Newnes.

11. I. Boldea, S. A. ( 2005). Linear Electric Actuators and Generators (illustrated, reprint ed.). Cambridge , United Kingdom : Cambridge University Press.

12. Minsoo Kim, S. A. (2003). An Adaptive Beamforming Algorithm. Texas, USA.

13. Molisch, A. F. (2010). Wireless Communications (2 ed.). West susex , united Kingdom: John Wiley \& Sons.

14. Rao, A. S. (1947). Electronic Device for Plotting Antenna Radiation Patterns. California, USA: Stanford Universi 